\title{
An Interactive Layers Model of Self-Regulated Learning and Cognitive Load
}

\author{
Joachim Wirth ${ }^{1}$ (D) Ferdinand Stebner ${ }^{1}$ (D) Melanie Trypke ${ }^{1} \cdot$ Corinna Schuster $^{2}$ (D) \\ Detlev Leutner ${ }^{3}$
}

Published online: 10 September 2020

(C) The Author(s) 2020

\begin{abstract}
Models of self-regulated learning emphasize the active and intentional role of learners and, thereby, focus mainly on conscious processes in working memory and long-term memory. Cognitive load theory supports this view on learning. As a result, both fields of research ignore the potential role of unconscious processes for learning. In this review paper, we propose an interactive layers model on self-regulated learning and cognitive load that considers sensory memory, working memory, and long-term memory. The model distinguishes between (a) unconscious self-regulated learning initiated by socalled resonant states in sensory memory and (b) conscious self-regulated learning of scheme construction in working memory. In contrast with conscious self-regulation, unconscious self-regulation induces no cognitive load. The model describes conscious and unconscious self-regulation in three different layers: a content layer, a learning strategy layer, and a metacognitive layer. Interactions of the three layers reflect processes of monitoring and control. We first substantiate the model based on a narrative review. Afterwards, we illustrate how the model contributes to re-interpretation of inconsistent empirical findings reported in the existing literature.
\end{abstract}

Keywords Consciousness $\cdot$ Metacognition $\cdot$ Resonant states $\cdot$ Sensory memory

\section{Introduction}

Self-regulated learning means that learners actively assume personal responsibility for their learning (Zimmerman 2000) and, therefore, decide whether, what, when, how, and why they

Joachim Wirth

lehrlernforschung@ rub.de

1 Ruhr University Bochum, Universitätsstraße 150, 44801 Bochum, Germany

2 University of Münster, Münster, Germany

3 University of Duisburg-Essen, Essen, Germany 
learn (Veenman et al. 2006; Weinert 1982). Numerous models have been established to describe ideal processes of self-regulated learning (for an overview, see Panadero 2017; Puustinen and Pulkkinen 2001; Winne and Perry 2000; Zimmerman 2008). These different models share at least two core assumptions. First, self-regulated learning is a highly complex process. It simultaneously engages cognitive, metacognitive, motivational, and behavioral processes (Boekaerts 1997; Winne and Hadwin 1998). These processes interact with one another, and they show a cyclic nature in which learners continuously use feedback to adjust their learning (Leopold and Leutner 2015; Schneider and Pressley 1989; Zimmerman 2008). Second, self-regulated learners engage consciously in the processes of planning, goal setting, organizing, monitoring, and evaluating. To that end, self-regulated learners consciously choose and use cognitive, metacognitive, motivational, and behavioral learning strategies, continuously monitoring learning progression in terms of quantity and quality (Glogger et al. 2012; Wirth and Leutner 2008).

The assumption of conscious engagement, however, must be queried for several reasons. One reason is that, according to models of cognitive processing, such as Atkinson and Shiffrin's (1968) structural component model, consciousness is limited to a small number of processes at a time. Conscious processes take place in short-term memory, which Baddeley and Hitch (1974) call "working memory." However, working memory capacity is limited in both the capacity to store information and the capacity to process information (Sweller 1988). Therefore, learners can consciously perform only a limited number of processes at the same time (Kuldas et al. 2013; Rhodes and Cowan 2018). Given the complexity of self-regulation of learning, one may question how self-regulated learners can consciously engage in all the different cognitive, metacognitive, motivational, and behavioral regulation processes. A second reason derives from intervention studies that show that learners improve in self-regulation through training, especially when training occurs over a long period of time and learners frequently repeat the use and regulation of learning strategies (De Boer et al. 2018). This observation is in line with skill acquisition theory, developed by Anderson (1982), which proposes that learners proceduralize skills through repeated practice. One effect of proceduralization is that the skill is performed automatically and autonomously. On the one hand, this reduces the load on working memory. On the other hand, it also reduces the need for conscious control.

Given the complexity of self-regulated learning and the limitations of working memory, one can assume that learners are not usually able to consciously self-regulate all learning processes at the same time. Being aware of all cognitive, metacognitive, motivational, and behavioral learning processes and regulating each of these processes consciously would inevitably lead to cognitive overload. Therefore, we conclude that models of self-regulated learning should not rely solely on an active and conscious kind of self-regulation. We propose that models of self-regulated learning need to include processes of self-regulation that are also reactive and unconscious. Since unconscious self-regulation may occur without inducing cognitive load, it might be one reason to think of self-regulated learning as a complex process that does not necessarily lead to cognitive overload.

In this paper, we propose a model of self-regulated learning and cognitive load that incorporates four main assumptions. First, it represents the complexity of self-regulated learning by proposing three different layers that reflect the different processes on a (learning) content level, a learning strategy level, and a metacognitive level (cf. Boekaerts 1997). Second, the model represents the following three structural memory components proposed by Atkinson and Shiffrin (1968): sensory memory, short-term memory (or 
working memory; Baddeley and Hitch 1974), and long-term memory (Mayer 1997). Third, layers and components interact in that they exchange information and monitor and control each other (Nelson and Narens 1990). Fourth, processes of self-regulation are not restricted to working memory but are additionally initiated in sensory memory. According to this assumption, substantial parts of the self-regulation of learning are performed without conscious awareness (Kuldas et al. 2013). This assumption is not new. For example, Pressley et al. (1987, 1989) proposed that good strategy users or good information processors can use strategies consciously or habitually (see also De Lisi 1987) as long as they experience no obstacles. However, their model does not describe how the use of this unconscious selfregulated strategy might take place. To clarify this process, we propose a mechanism derived from Adaptive Resonance Theory (ART; Grossberg 1976a, b, 1999, 2019), which explains how information can be processed automatically without reaching working memory as long as the information is consistent with expectations derived from long-term memory.

In the following paragraphs, we will first describe the mechanism proposed for sensory memory. Afterwards, we will discuss how learning takes place in the different layers, given the proposed mechanism in sensory memory. We will then describe how the different layers interact. Furthermore, we will present empirical findings of research on self-regulated learning, metamemory, and cognitive load theory and show how the proposed model helps to better understand the reported empirical findings. Finally, for validation purposes, we will derive and present new hypotheses to test the model empirically in future research.

\section{Unconscious Self-Regulation}

Grossberg (1976a, b, 1999, 2019) proposed ART to solve a problem he calls "stabilityplasticity dilemma" (Grossberg 1999, p. 3). The dilemma derives from the necessity to rapidly learn a vast amount of information in a rapidly changing environment without forgetting what has already been learned. To solve this problem, Grossberg proposes the so-called resonant states, and he assumes that resonance is necessary — but not sufficient - for consciousness (cf. Bor and Seth 2012; Cohen et al. 2012; Pitts et al. 2018). In short, resonance is a result of the interaction of a bottom-up and a top-down process. Single sensory information or information patterns of the environment or of the individual enter the cognitive system in a bottom-up process. They can activate expectations retrieved in a top-down process from long-term memory, for example, in the form of schemes. Additionally, an expectation might be primed beforehand. If sensory information and expectation match, they amplify each other, resulting in resonance. Sensory information in a resonant state lasts longer and is more energetic than sensory information not amplified by a bottom-up top-down interaction. If the resonant state is strong enough and lasts long enough, it achieves consciousness.

Of course, not all sensory information matches with expectation. Most mismatched sensory information will decay. However, if sensory information is strong enough without amplification by expectation, it may trigger a long-term memory search for a more appropriate scheme that helps to interpret mismatched information, resulting in a resonant state.

We propose a mechanism akin to Grossberg's resonant state resulting from bottom-up and top-down interaction for subliminal information processing located in sensory memory. When sensory information enters sensory memory, it needs to receive attention for further processing (Atkinson and Shiffrin 1968; Cohen et al. 2012). Otherwise, the information will decay after several hundred milliseconds. The mechanism of resonant states suggests that attention results 
from the interaction of sensory information and long-term memory information and is expressed by resonance. Resonance is proposed to increase the likelihood that information is processed consciously. In other words, resonance increases the likelihood of information's being transferred from sensory memory to working memory (cf. Sweller et al. 1998).

However, resonance does not automatically lead to sensory information's achieving consciousness (Grossberg 1999). Resonant states might stay unconscious; that is, resonant states might stay in sensory memory. This means, accordingly, that if attention is expressed by resonance and resonant states can remain unconsciously in sensory memory, sensory information can receive attention without achieving consciousness and being transferred to working memory. As a consequence, when sensory information is part of a resonant state that stays in sensory memory, it interacts with long-term memory information and, therefore, can enter long-term memory and contribute to learning without being transferred to working memory (e.g., Cowan 1997, 2008). The concept of resonant states explains how information can contribute to learning without being transferred to working memory, without achieving consciousness, and without inducing cognitive load on working memory.

Resonance results from the interaction of bottom-up sensory information and top-down long-term memory information amplifying each other. This is likely to happen if sensory information matches long-term memory information. However, what if sensory information does not match long-term memory information? If sensory information is weak, it will decay. If sensory information is strong enough and lasts long enough, though, it is likely to be transferred to working memory, triggering a long-term memory search for information that matches the sensory information. This way, mismatched information (e.g., surprising information) receives not only attention but also conscious awareness and, thus, induces cognitive load (cf., Frensch and Rünger 2003).

An example may best illustrate this mechanism. When driving a car, you need to process a large amount of information at any point in time. Most of the information does not achieve consciousness. Some of the information is irrelevant - for example, the color of the car behind you - and will decay without achieving consciousness. Other information is relevant but also does not achieve consciousness. For instance, in most cases, keeping the car on track is an automatic process of turning the steering wheel, and you are not aware of turning the steering wheel at all. This automatic, unconscious but relevant process can be explained by resonant states. Sensory information (received by your eyes, hands, ears, and so forth) aligns with your expectation of the car's being on track. However, as long as the road is straight and nothing surprising happens, resonance will not be strong enough to transfer information to working memory. In other words, steering the car remains a collection of unconscious processes initiated in sensory memory, not transferred to your working memory and, therefore, not inducing cognitive load. As a result, you are able, for example, to actively engage in a conversation with your passenger. Imagine you reach a construction area, however. Your lane becomes narrow and curvy, and a considerable number of traffic signs guide you through an unusual route. Now your sensory information no longer matches your expectation of a straight road. It may transfer to working memory and trigger long-term memory search in order to interpret the traffic signs. These search and interpretation processes induce cognitive load. As a consequence, you will likely stop talking and concentrate on the road and traffic signs since there remains no sufficient working memory capacity for engaging in an intensive conversation.

We assume that the concept of resonant states as a mechanism of sensory memory can explain how learners are able to self-regulate the complex interaction of all the cognitive, 
metacognitive, motivational, and behavioral processes that are involved in learning and take place at the same time, without overloading working memory. The core assumption is that resonant states combine sensory information with long-term memory information and, therefore, contribute to learning without necessarily achieving consciousness and being transferred to working memory, inducing cognitive load. We call this kind of process "unconscious selfregulation," which is unconscious insofar as it is automatic rather than intentional. However, the process is still self-regulation since the learner still regulates the learning instead of others guiding it. We assume that this mechanism works on all layers of self-regulated learning: the content layer, the learning strategy layer, and the metacognitive layer (Boekaerts 1997). We will elaborate on this within the following sections.

\section{Content Layer}

Content learning aims at the construction of schemes (Abelson 1981; Bartlett 1932; Chi et al. 1982; Koedinger and Anderson 1990). Schemes are knowledge structures stored in long-term memory. They are highly efficient for several reasons. They can contain a significant amount of information in an organized manner, retrieved to working memory as one or just a few information units. In addition, they guide the learner's perception of what is relevant and help the learner ignore irrelevant information. Schemes can house different knowledge types and interrelate different knowledge types within a single scheme. Depending on the type of knowledge, scheme acquisition differs. Learners construct schemes that represent concepts and facts by selecting, organizing, and elaborating on information as well as by combining new information with prior knowledge so that, in the best case, learners arrive at a deep understanding of the concepts or facts to be learned (Mayer 1997). In contrast, learners acquire schemes representing knowledge about procedures through frequent rehearsal and practice. Anderson (1982) theorized processes of composition and proceduralization that lead to procedural steps and procedures being chunked into one coherent scheme. Of course, schemes might also comprise both: knowledge about concepts and facts and knowledge about procedures. For example, a certain learning strategy's scheme not only entails knowledge about single action steps (procedural knowledge; "then-part" of a production according to Anderson 1982) but also entails an understanding of why the learning strategy is useful in certain learning situations (conceptual knowledge; "if-part" of a procedure). As a consequence, the acquisition of schemes comprising different types of knowledge might require the combination of different learning strategies.

The acquisition of schemes usually requires working memory capacity (Fig. 1). Scheme construction is often described as transfer of relevant sensory information as well as retrieval of long-term memory information into working memory, where generative learning processes of organization, elaboration, and rehearsal construct and modify schemes. These learning processes are conscious, inducing cognitive load on working memory (Sweller 1988; Sweller et al. 1998, 2019). They refer to explicit learning (Reber et al. 1991), where learners intentionally engage in learning.

However, assuming a mechanism, such as resonant states in sensory memory, encourages one to describing scheme acquisition that is not intentional and conscious and that, therefore, might relate to implicit learning (Berry and Dienes 1993; Cleeremans 1993; Reber et al. 1991; Stadler and Frensch 1998). Implicit learning is often described as incidental learning (Dienes and Berry 1997): a learning process that occurs without being intentionally initiated by the learner. Early studies on implicit learning of artificial grammar by Reber (1967) reveal that 


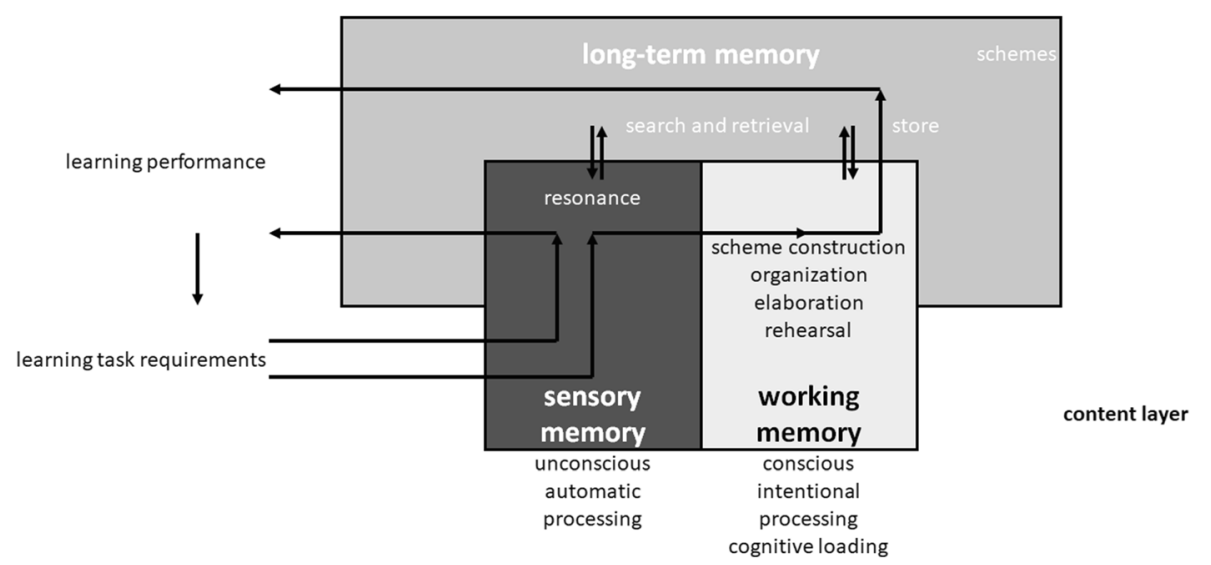

Fig. 1 Content layer of the interactive layers model of self-regulated learning and cognitive load (top view)

implicit learning depends on a high frequency of learning events presented one after another. This high frequency of repeated learning events makes it possible for learners to unconsciously form expectations based on earlier learning events because previous events' sensory information is still present in sensory memory when the next learning event's information enters sensory memory. When sensory information of a current learning event matches sensory information of a previous learning event, resonance emerges, which in turn increases the likelihood that the information enters long-term memory and contributes to inductive scheme acquisition (e.g., Stadler 1992). The resulting scheme may be very simple at the beginning of learning, representing just a single association (Broadbent et al. 1986; Churchill and Gillmore 1998). If in subsequent learning events learners retrieve this scheme to sensory memory, however, new resonant states with new sensory information can occur, which in turn results in an increasingly sophisticated scheme structure. By this, even complex structures, such as artificial grammar, can be learned without consciousness and without inducing cognitive load. It must be noted that learners are not aware of implicitly learned schemes and, as a consequence, do not generate an understanding of what they have learned. As Reber (1967) has demonstrated, learners can use implicitly learned schemes to more rapidly learn stimuli that correspond to the scheme though they are unable to verbalize the knowledge represented by the scheme. Thus, it must be distinguished between representing knowledge by a scheme and understanding the structure of the scheme. Learners can apply implicitly learned schemes without having developed an understanding of the structure of the scheme (e.g., Hayes and Broadbent 1988; Stanley et al. 1989). Implicitly learned schemes might be seen as a complex network of associations that can affect learning and behavior without consciousness and understanding. In that way, they differ from schemes that learners consciously construct in working memory and that lead to more or less deep understanding (Koedinger et al. 2012).

In summary, we propose two ways of learning within the content layer. One way is conscious, intentional, and induces cognitive load. Schemes as well as sensory information that is part of a strong and long-lasting resonant state in sensory memory are retrieved into working memory. In this case, conscious learning indicates constructing and modifying schemes by organization, elaboration, and rehearsal in working memory, inducing cognitive load. The second way is unconscious and does not induce cognitive load. Although incidental, this kind of learning is not random. Sensory information matches expectation based on 
previous learning events or activated schemes. The more often sensory information is in resonance with expectations, the more likely its transfer to long-term memory, either building a new scheme or modifying an existing scheme without working memory being involved and, thus, without inducing cognitive load.

\section{Learning Strategy Layer}

Learning strategies are at the core of self-regulated learning. We distinguish cognitive, metacognitive, motivational, and resource management strategies (Engelschalk et al. 2017; Pintrich and De Groot 1990; Waldeyer et al. 2019; Weinstein and Mayer 1986; Wolters 1998), which all express themselves in strategic learning behavior. As mentioned earlier, all models of self-regulated learning describe self-regulated learners as consciously organizing their learning by selecting and applying learning strategies based on task requirements and conditions (e.g., instructional cues, available resources), strategy knowledge and cognitive conditions (e.g., prior knowledge, beliefs), and personal objectives (Winne and Hadwin 1998). To accomplish this effectively, learners need declarative, procedural, and conditional strategy knowledge (Paris et al. 1983). Declarative strategy knowledge refers to knowing "that" and includes knowledge about the learning strategy that can be verbally explained to others. Procedural strategy knowledge refers to knowing "how" to perform a certain learning strategy and all the actions involved. Finally, conditional strategy knowledge refers to knowing "why" and "when" and "under which conditions" a certain learning strategy is applicable and useful for learning.

Intervention studies have demonstrated that learners can acquire learning strategy knowledge and improve their use of learning strategies (e.g., Aghaie and Zhang 2012; Eilers and Pinkley 2006; Leopold and Leutner 2015). A typical intervention starts with verbally explaining the strategy and its different steps and demonstrating its use. Afterwards, learners have repeated opportunities to apply their acquired learning strategy knowledge to different but similar learning tasks (e.g., Leopold and Leutner 2015). This way, learning strategy interventions typically include phases of skill acquisition as described by Anderson (1982). In the declarative phase, learners are asked to interpret all the single features of the learning task as well as each step of the strategic procedure. They need to understand the structure of the type of task as a condition for the successful use of the respective learning strategy ("if-part" of a production according to Anderson 1982). Additionally, learners need to know the action steps of the learning strategy ("then-part") and integrate them into the to-be-built learning strategy scheme ("production"). In this declarative phase, learning strategy knowledge is conscious and the acquisition of learning strategy knowledge, therefore, induces cognitive load. Learning strategy knowledge is also declarative in this stage, with both teachers and learners able to declare and verbalize it so that knowledge is mediated verbally. Note that declarative knowledge, as defined by Anderson (1982), holds for all three kinds of learning strategy knowledge theorized by Paris et al. (1983), not only for knowing "that." Declarative knowledge sensu Anderson is different from declarative knowledge sensu Paris et al. (Süß 1996). Declarative knowledge sensu Anderson indicates that knowledge can be or needs to be declared and verbalized, while declarative knowledge sensu Paris et al. denotes conceptual knowledge (knowing "that").

In the phase of knowledge compilation, learners practice learning strategy use repeatedly and, thereby, convert formerly declarative knowledge (sensu Anderson) into proceduralized knowledge. With increasing proceduralization the need for consciously interpreting single 
conceptual information of the learning strategy (conceptional and conditional knowledge) decreases, and single action steps of the learning strategy are composed into productions and macroproductions. As a result, cognitive load induced by the use of a learning strategy also decreases. In the final phase, the procedural phase, further tuning of the acquired learning strategy scheme is achieved by generalization, discrimination, and strengthening. These processes especially affect conditional strategy knowledge so that learners easily recognize under which conditions a learning strategy is applicable; therefore, deciding which learning strategy to use induces less cognitive load.

Thus far, we have described how learners acquire learning strategy knowledge and become experienced users of learning strategies. Next, we will describe two ways of using learning strategies when performing a certain learning task (Fig. 2). Again, these two ways differ in the amount of cognitive load they induce on working memory. The way usually described by models of self-regulated learning starts with the learning task and its conditions for learning. These conditions include affordances for certain learning strategies. For example, learning and understanding a science text might prompt using deep learning strategies of organization and elaboration (e.g., concept mapping, summarizing, or visualizing; Leopold and Leutner 2015) instead of rehearsing the text literally. In contrast, vocabulary might efficiently be learned by rehearsal, establishing association between the word of the mother tongue and the word of the foreign language. Thus, learning task affordances might prompt certain learning strategies. However, learners need to consciously decide which learning strategy to use not only based on task affordances but also on their learning-strategy knowledge and their learning objectives (Winne and Hadwin 1998). They need to retrieve and consciously process all this information in working memory, thereby experiencing cognitive load.

However, learners do not always think intensively about which strategy to use when they begin with learning. One may argue that this kind of learning is not strategic and self-regulated at all. One may also argue that, in some cases, learners - especially good strategy users (Pressley et al. 1987, 1989) — possess strong learning-strategy knowledge that is activated in a top-down process when entering the learning situation. In that case, information regarding task affordances may, in a bottom-up process, enter sensory memory and match conditional learning-strategy knowledge, resulting in resonance. This resonance may unconsciously initiate learning-strategy application. This way, learners can perform learning strategies and selfregulate their learning without continuously thinking about how to perform the strategy or

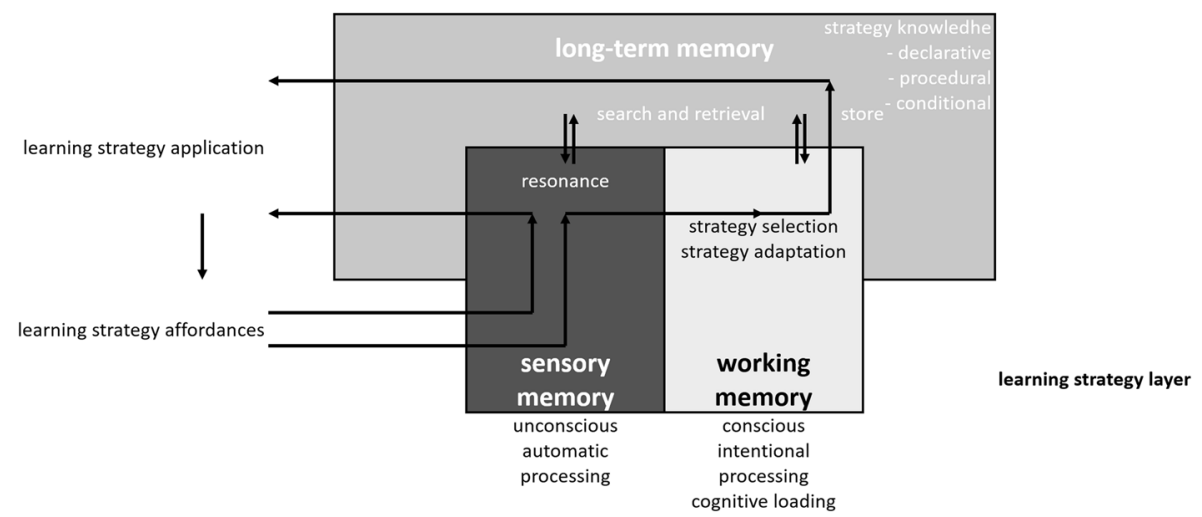

Fig. 2 Learning strategy layer of the interactive layers model of self-regulated learning and cognitive load (top view) 
about whether this learning strategy is the best. Thus, learning can be strategic and selfregulated without inducing cognitive load on working memory.

\section{Metacognitive Layer}

Monitoring and control are the two key processes of self-regulated learning that are based on metacognition (Butler and Winne 1995; Nelson and Narens 1990; Winne 1996). Self-regulated learners monitor their learning with respect to two different aspects (Wirth and Leutner 2008). One, they evaluate whether they meet their learning goals (content layer). And two, they evaluate whether they have executed a learning strategy the way they had planned (learning strategy layer). In case of discrepancies with respect to goals or strategy, self-regulated learners control their learning by adjusting their learning behavior. In the following, we use the term metacognitive regulation to refer to the interplay of monitoring and control.

Flavell (1979) described monitoring as an interaction of metacognitive knowledge, metacognitive experiences, learning goals, and learning strategies, whereby learning goals and learning strategies may be set and chosen based on metacognitive knowledge and metacognitive experiences. Metacognitive knowledge is a learner's knowledge, stored in long-term memory, about characteristics of the learner or other persons, of the learning task, and of learning strategies (Fig. 3). Metacognitive person knowledge includes knowledge about intra- and interindividual differences on cognitive processes as well as beliefs about universal properties of cognition. Metacognitive task knowledge concerns information available during task performance as well as typical demands and goals of a specific type of learning task. Metacognitive learning strategy knowledge includes declarative, procedural, and conditional strategy knowledge as described above. When using these three kinds of metacognitive knowledge, learners should be aware that they may depend on each other. For example, metacognitive learning strategy knowledge and metacognitive person knowledge interact in such a way that learners not only know certain learning strategies but also know whether they are capable of applying a certain learning strategy or not.

Flavell (1979) argued that learners may activate metacognitive knowledge consciously, resulting in metacognitive experience. Metacognitive experiences occur in situations that require careful and conscious thinking. For example, when learners realize that a learning task is difficult, they engage in standard setting (Winne and Hadwin 1998) and planning in

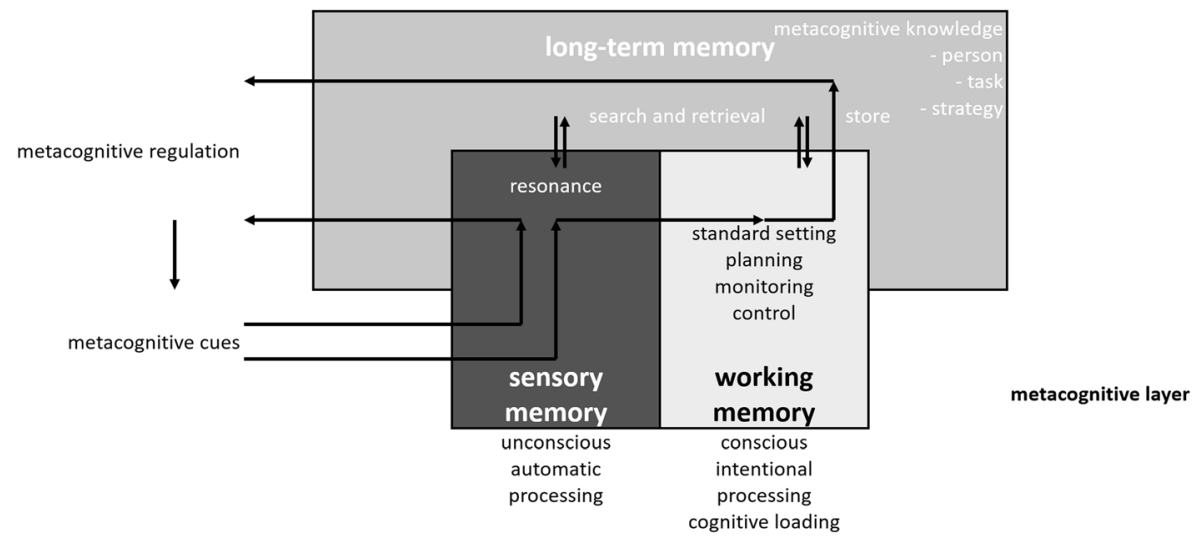

Fig. 3 Metacognitive layer of the interactive layers model of self-regulated learning and cognitive load (top view) 
order to master the task. In that case, learners retrieve metacognitive knowledge into working memory and use it for metacognitive regulation of their learning. However, according to Flavell (1979), metacognitive knowledge may also be activated by metacognitive cues in the task situation without forming a metacognitive experience (cf. Evans 2006; Kahneman 2003; Sloman 1996). Metacognitive knowledge can then influence learning without itself entering consciousness (Baker 1994; Reder 1996; Veenman et al. 2002). We assume that this kind of unconscious self-regulation is likely to happen if metacognitive cues match metacognitive knowledge and cause resonance in sensory memory. This resonance may be strong enough to directly contribute to metacognitive regulation. At the same time, the resonance may not be strong enough to transfer into working memory and become a conscious metacognitive experience. For instance, imagine reading a text. If you expect yourself to able to easily understand the text (since you have often read this kind of text, you know how to read this kind of text, you have sufficient prior content knowledge, etc.), you will probably start reading the text in the way you usually read this kind of text without engaging in careful and conscious planning and metacognitive regulation (Kintsch 1998). As long as you read fluently through the text, monitoring and control may remain an unconscious process based on resonant states in sensory memory (Koriat et al. 2014; Samuels et al. 2005). However, imagine encountering a paragraph that is surprisingly difficult to understand. In this case, metacognitive cues, such as approaching unknown vocabulary or slowing down reading speed, may grow strong enough to transfer to working memory, even without being amplified by resonance. As a result, these metacognitive cues become part of a conscious metacognitive experience. Thus, in this case, metacognitive regulation becomes conscious and induces cognitive load.

\section{Layer Interaction}

In the previous sections, we have described self-regulated learning as processes in three different layers: content layer, learning strategy layer, and metacognitive layer. All three layers include the same structural components of sensory memory, working memory, and long-term memory. For all three layers, we assume the same kind of cognitive processes. Sensory information enters sensory memory. And if it matches long-term memory information, it creates a resonant state. If resonance is strong enough, sensory information automatically contributes to learning and its (unconscious) regulation without achieving consciousness and without inducing cognitive load. If the resonance becomes even stronger, it transfers to working memory, where it is consciously and intentionally processed, resulting in cognitive load. If there is a incompatibility of sensory information and long-term memory information, and if the sensory information itself is strong enough, sensory information transfers to working memory as well.

Although self-regulated learning is a highly complex interaction of structural components and cognitive processes within each layer, it may be even more complex since we additionally assume interactions across the three layers (Fig. 4). These interactions of self-regulated learning may be part of an intentional and conscious process in working memory (interactions 6 to 10 in Fig. 4), inducing cognitive load (e.g., Valcke 2002). They can also occur, however, within an automatic and unconscious process in sensory memory without inducing cognitive load (interactions 1 to 5 in Fig. 4). We will start with describing intentional, conscious, and cognitive load-inducing working memory interactions of self-regulated learning. 


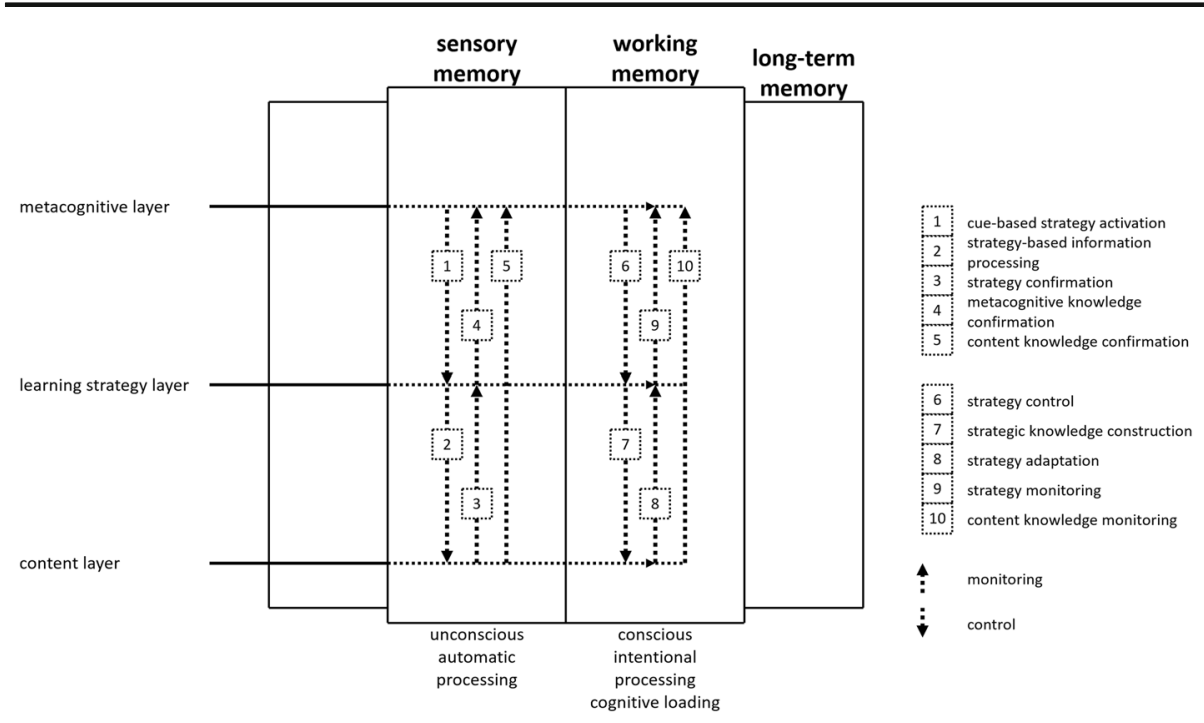

Fig. 4 Layer interaction of the interactive layers model of self-regulated learning and cognitive load (front view)

Layer interactions occur as top-down and bottom-up interactions, representing Nelson and Narens' (1990; Nelson 1996) processes of monitoring (bottom-up) and control (top-down). For example, judgments of learning as part of the monitoring process result from a bottom-up interaction of the content layer and the metacognitive layer (interaction 10 in Fig. 4). Judgments of learning are "predictions about future test performance on currently recallable items" (Nelson and Narens 1990, p. 130). For these conscious kinds of metacognitive judgments, learners need to rely on information of the content layer stored in working memory and evaluate this information in order to up-date their metacognitive person knowledge. If learners realize a gap in content knowledge, control processes may start (e.g., Komori 2016). Learners need to select an appropriate learning strategy from their metacognitive learning strategy knowledge (interaction 6 in Fig. 4) and adapt it to the current learning task (interaction 7 in Fig. 4) in a top-down process. Through a bottom-up interaction of the learning strategy layer and the content layer, learners monitor whether their adaptation of learning strategy knowledge to the current learning task is successful (interaction 8 in Fig. 4) or whether further adaptation is possible and needed (top-down interaction 7 in Fig. 4). On this basis, learners conclude whether they have chosen an appropriate learning strategy and whether they have implemented this learning strategy with sufficient quality (bottom-up interaction 9 in Fig. 4). This interaction of the learning strategy layer and the metacognitive layer will refine declarative, procedural, and conditional knowledge of the learning strategy layer as well as metacognitive learning-strategy knowledge on the metacognitive layer.

Of course, this is a description of an ideal, successful layer interaction and successful selfregulation of learning. However, self-regulation is not always successful. Students may stop studying too early or may use an inappropriate learning strategy (e.g., Mazzioni et al. 1990). According to our model, unsuccessful self-regulation results from deficits of at least one of the layer interactions. For example, judgments of learning might be not perfectly calibrated because of deficient content knowledge monitoring (interaction 10 in Fig. 4). Deficient content knowledge monitoring might be due to a deficient representation of relevant content knowledge in working memory or due to deficient retrieval of metacognitive knowledge. Learners 
might need feedback or prompts (Wirth 2009) to recognize the deficient layer interaction and the reasons for this in order to improve their self-regulation in the next learning cycle.

In addition to the intentional and conscious interactions in working memory, we postulate that similar interactions take place in sensory memory and, thus, occur as automatic processes that do not induce cognitive load on working memory. This assumption is in line with dualprocess theories (Evans 2006; Kahneman 2003; Sloman 1996), which distinguish an automatic, "intuitive" type 1 process (Thompson et al. 2011) from a deliberate, analytic, "intentional" type 2 process. Monitoring of type 1 processes uses implicit cues (Koriat 2007), such as the fluency of cognitive processes, and may result in a feeling of rightness (Thompson 2009). Strong feelings of rightness, in turn, prevent learners from engaging in type 2 processes.

We argue that, in self-regulated learning, type 1 processes occur as unconscious interactions in sensory memory. They can be explained by resonant states of one layer that amplify a resonant state of another layer. One layer's resonant states may amplify another layer's if the resonances match and swing in accordance with each other. Thereby, resonant states of one layer can confirm or even activate resonant states of another layer.

The feeling of knowing, as part of monitoring, may be one example of such an automatic and unconscious interaction of resonant states of different layers: "Feeling-of-knowing (FOK) judgments occur during or after acquisition (e.g., during a retention session) and are judgments about whether a given currently nonrecallable item is known and/or will be remembered on a subsequent retention test" (Nelson and Narens 1990, p. 130). As such, these metacognitive judgments refer to nonrecallable information of the content layer; this information cannot currently be retrieved from long-term memory to working memory. In a case of FOK, a resonant state of the content layer may amplify a resonant state of the metacognitive layer (bottom-up interaction 5 in Fig. 4) so that the learner experiences the feeling of the resonance established by sensory information and long-term information on the content layer. This feeling can confirm the metacognitive monitoring judgment of a learner on the metacognitive layer, and the learner experiences FOK of content without consciously being aware of this content.

An example of an automatic, unconscious control process may be reading a page with a few words highlighted in italics. These metacognitive cues might match a learner's metacognitive task knowledge about highlighted words in a text being likely relevant for understanding the text, resulting in a resonant state in the metacognitive layer. This resonance on the metacognitive layer may amplify the perception of the highlighted words as learning strategy affordances, which may confirm learning strategy knowledge about first skimming highlighted words before reading the entire page (top-down interaction 1 in Fig. 4). Activating this reading strategy amplifies the content information of the highlighted words, which in turn are more likely to create a resonant state with respect to prior knowledge on the content layer (top-down interaction 2 in Fig. 4). When sensory information matches long-term memory information (prior knowledge) on the content layer, the resulting resonance may amplify the respective resonant state on the learning strategy layer and, thereby, confirm the reading strategy of first skimming highlighted words (bottom-up interaction 3 in Fig. 4). If there is a mismatch and if sensory information of a highlighted word is strong enough to transfer to working memory (that is, this information is perceived as sufficiently relevant), it may trigger a conscious search for appropriate prior knowledge or may activate conscious monitoring and control processes in working memory. As long as learners encounter no obstacles when skimming the text and activating prior knowledge, the resonant state on the learning strategy layer persists and may amplify the respective resonant state on the metacognitive layer (bottom-up interaction 4 in 
Fig. 4). In effect, the metacognitive task knowledge about highlighted words as being a text's relevant information is confirmed.

In summary, Fig. 5 illustrates the proposed interactive layers model of self-regulated learning and cognitive load. The model consists of the three structural components (sensory memory, working memory, and long-term memory), three layers (content layer, learning strategy layer, and metacognitive layer), and interaction processes of structural components within one layer as well as interaction processes between different layers. It distinguishes intentional and conscious processes of self-regulated learning from more intuitive and unconscious self-regulated learning processes. Conscious processes are located in working memory and, therefore, induce cognitive load. Research on self-regulated learning mainly investigates these conscious self-regulation processes (e.g., Azevedo et al. 2008; Nückles et al. 2009). Research on perception and cognition, however, suggests unconscious processes in addition to conscious processes of self-regulated learning (Runeson et al. 2000). In our model, these unconscious processes are located in sensory memory and explained by resonant states that allow sensory information to interact with long-term information without achieving consciousness while still contributing to learning. Consequently, these resonant states contribute to selfregulated learning without inducing cognitive load. This study argues that resonant states and resulting processes are part of self-regulation of learning, although self-regulated learners are not aware of these processes. Resonant states and resonant state interactions are processes that attend to certain sensory information as well as certain long-term information. They process and refine long-term information and can lead to strategic and metacognitively regulated information processing. Therefore, they share the same core features as conscious processes of self-regulated learning, excluding awareness and consciousness, which contributes to selfregulated learning without inducing cognitive load. We call this kind of automatic processes unconscious self-regulated learning.

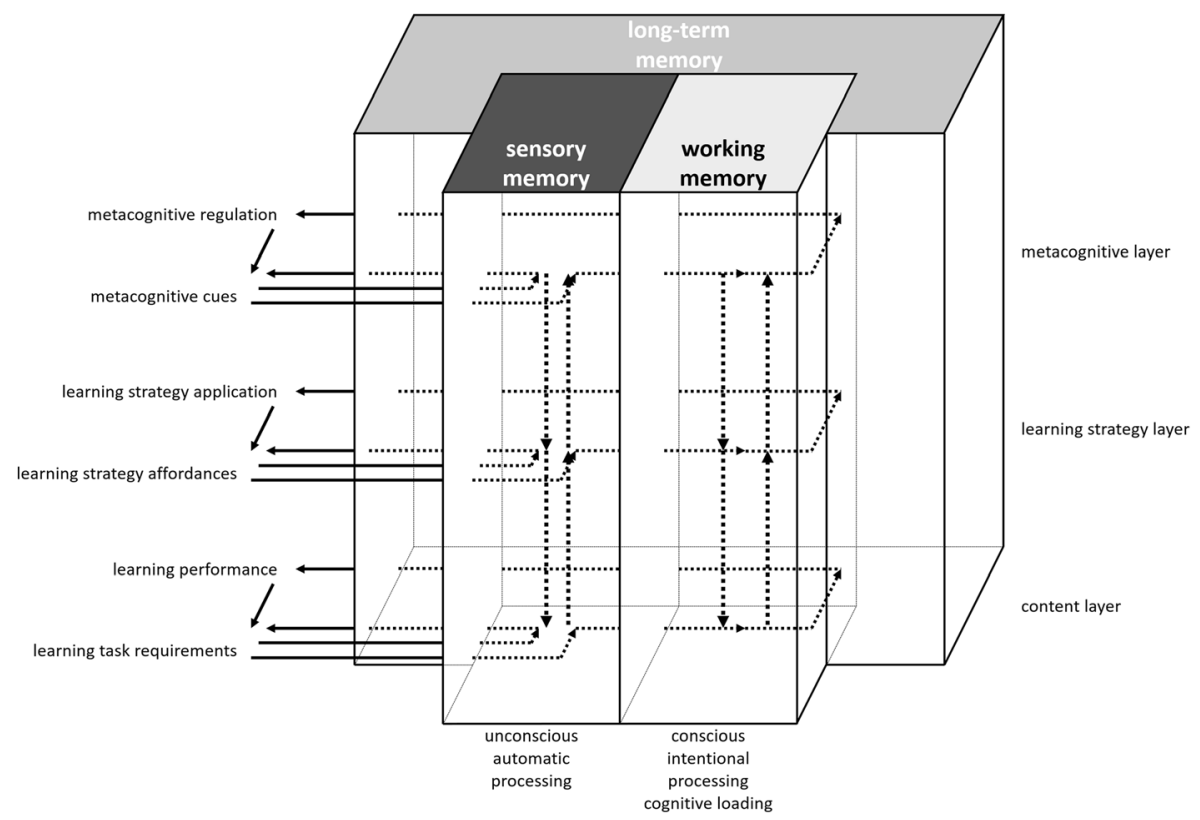

Fig. 5 Interactive layers model of self-regulated learning and cognitive load 


\section{Re-interpretation of Empirical Findings}

Proposing a new model, such as the interactive layers model of self-regulated learning and cognitive load, contributes to existing research only if the new model explains empirical findings that have been difficult to interpret or explain. In the following sections, we will report some empirical findings from the field of self-regulated learning, metacognitive judgements, and cognitive load theory that are difficult to explain by theories the studies' authors consulted. We will show how the interactive layers model assists in interpreting these findings.

\section{Findings on Self-Regulation of Learning and Learning Outcome}

According to models of self-regulated learning, self-regulation contributes to learning. As a consequence, researchers expect positive correlation between self-regulation and learning outcome (e.g., Wang et al. 1990). However, empirical research on the correlation of selfregulation and learning outcome reveals mixed results (Blickle 1996; Pintrich and Garcia 1993; Pintrich et al. 1993; Veenman et al. 2005). While there are studies finding positive correlations, as expected, others find weak or no correlation.

These inconsistent results appear not only across studies but also within studies. For example, Leopold and Leutner (2002) compared students of different grades and expected to find higher correlations of self-regulation of learning and learning outcome in higher grades compared with lower grades. Lower-grade students are not experienced in self-regulated learning and thus cannot effectively self-regulate, whereas higher-grade students have had many opportunities to develop self-regulated learning skills, resulting in variance in effective self-regulated learning between students. In Leopold and Leutner's (2002) study, students of different grades read an expository science text. Afterwards, they were asked about their use of different learning strategies and were tested on text comprehension. For cognitive deep-level learning strategies, results were consistent with expectations. Correlations of the use of deeplevel learning strategies and text comprehension increased from $r=.01$ (grade 5) to $r=.29$ (grade 7) and from $r=.39$ (grade 9) to $r=.44$ (grade 11), following a monotone trend. However, correlations of the use of metacognitive learning strategies and text comprehension were surprising since they followed a non-monotone trend, increasing from $r=-.05$ (grade 5) and $r=.23$ (grade 7) to $r=.31$ (grade 9) before decreasing to $r=.15$ (n.s., grade 11). For an explanation of these different result patterns, one must know that the survey questions covering deep-level learning strategies contained very specific information from the expository text. For example, one item asked, "Did you think about the relationship between the terms dipole and hydrogen bridge bond (no/ if anything, no/if anything, yes/yes)?" Students of all grades had no prior knowledge of the content of the expository text. Thus, according to the interactive layers model, we gather that students' low prior content knowledge resulted in a very low probability for resonant states on the content layer. Moreover, one may conclude that scheme construction required intentional and conscious working memory processes. Since deep-level learning strategy items contained specific content information, students' answers on these items also heavily relied on learning strategies intentionally used for scheme construction in working memory. Thus, these conscious processes were processes typically described by models of self-regulated learning and, accordingly, showed a result pattern consistent with these models. However, the result pattern of metacognitive strategy use was surprising; the correlation between reported metacognitive learning strategy use and learning outcome was lower for high-grade students than for middle-grade students. According to our model, this could be 
explained by a higher probability of resonant states on the metacognitive layer for experienced high-grade students. Of course, some high-grade students may have consciously used metacognitive knowledge, intentionally resulting in conscious metacognitive experiences. Other experienced students, however, may have unconsciously self-regulated their learning based on resonant states in sensory memory. Thus, we assume that, for less-experienced middle-grade students, self-regulation requires conscious processes in working memory. Notably, students can report whether they engaged in these processes or not. This may not be true for high-grade students since some unconsciously self-regulate their learning and, therefore, cannot report their self-regulation. Accordingly, for middle-grade students, correlations are consistent with models of self-regulated learning, while non-significant correlations for high-grade students indicate differences in conscious and unconscious self-regulation between high-grade students.

Of course, Leopold and Leutner (2002) is just one example of differences in correlational findings concerning metacognitive self-regulation and learning outcome, and our model provides just one of possibly many theoretical explanations of these differences. In our estimation, though, this example can inspire further research testing the assumption that selfregulation of learning can be conscious or unconscious, resulting in different correlations of self-regulation with learning outcomes.

\section{Findings on Different Measures of Self-Regulated Learning}

The study of Leutner and Leopold (2002) demonstrates that the validity of questionnaires administered after learning ("offline," Veenman et al. 2006; Wirth and Leutner 2008) depends on prior knowledge and learning experiences on all layers. Only when learners lack prior knowledge and self-regulation experiences, self-regulation relies mainly on working memory processes, which learners consciously experience and can validly report. However, if selfregulation induces too much cognitive load, it may be that validity of questionnaires may differ among the three layers. We assume that when learners experience risk of cognitive overload, they first concentrate on processes of the content layer since scheme construction is the primary task of learning. If some working memory capacity remains, learners engage in processes on the learning strategy layer and assign capacity to processes on the metacognitive layer. Thus, if the validity of questionnaires depends on working memory capacity invested in the respective layer, questionnaires on the content layers have a higher probability of validity than questionnaires on learning strategy layer. Similarly, questionnaires on the learning strategy layer are more likely to be valid than questionnaires on the metacognitive layer. These assumptions derived from the interactive layers model are confirmed and evidenced in the findings of a recent study by Rogiers et al. (2020). Through questionnaires and think-aloud protocols, Rogiers and colleagues measured students' cognitive and metacognitive strategy use when learning from texts (cf. Veenman et al. 2005, 2006). Students, averaging 13 years old, possessed low prior content knowledge and were categorized as average achievers by their teachers. Therefore, they correspond to the middle-grade students of the study by Leopold and Leutner (2002), and one might expect findings consistent with models of self-regulated learning. However, findings are inconsistent in many respects. For example, in the questionnaire, learners reported that they self-evaluated their learning frequently, but the authors did not observe any self-evaluation events in the think-aloud protocols. Additionally, the authors did not record any significant correlation between questionnaire self-reports on further metacognitive learning strategy use and respective think-aloud protocol data $(.10 \leq r \leq .11$, 
n.s.). In contrast, respective correlations concerning cognitive learning strategy use were moderate to strong $(.45 \leq r \leq .59)$. This pattern of results corresponds with Leopold and Leutner's findings and can be explained by our interactive layers model. As in Leopold and Leutner's study, probability for resonant states was low on all three layers, and self-regulated learning processes required conscious engagement and processing in working memory. Learners were able to report these processes on the learning strategy layer by thinking aloud and by completing the retrospective questionnaire. However, on the metacognitive layer, only a small number of utterances surfaced in the concurrent think-aloud data, and one must conclude that the learners did not engage in metacognitive monitoring and control. It seems that processes of self-regulated learning on the content layer and the learning strategy layer induced so much cognitive load that there was no working memory capacity left for conscious metacognitive processes. Since learners reported metacognitive strategy use in the retrospective questionnaire frequently, validity of these questionnaires must be queried. Answers may be attributed to social desirability.

\section{Findings on Metacognitive Accuracy and Learning Outcome}

Research on judgements of learning (JOL) indicates that JOLs are moderately accurate in predicting learning outcomes (Dunlosky and Nelson 1992; Koriat 1997) and that JOLs have an effect on study resources, such as study time that learners invest in learning (Son and Metcalfe 2000). One may expect that, with increased study time or with increased number of restudying trials, not only does learning performance increase but so does metacognitive accuracy since learners have better and more numerous opportunities to calibrate their metacognitive judgements, i.e., JOLs. However, Koriat et al. (2002) reported findings on JOLs that have shown that learners overestimate their learning after studying the material once but underestimate their learning when studying the learning material a second, third, or fourth time. Koriat and colleagues show that this "underconfidence-with-practice" (UWP) effect is independent from feedback, pacing, incentives, and other possible moderators. Inspecting the presented data reveals that the UWP effect results from different rates of learning performance and JOLs. While learning performance increases strongly with repeated restudying, JOLs increase only slightly.

As Koriat et al. (2002) point out, there is no convincing theoretical explanation for the UWP effect of JOLs, but the interactive layers model might be useful in this regard. The model proposes that JOLs rely on conscious interaction of the metacognitive layer and the content layer in working memory. Since the learning tasks in the study by Koriat et al. were "easy" (e.g., word pair associations), learners likely perceived these tasks as a simple kind of learning task. Thus, their metacognitive task knowledge may influence the interaction of content layer and metacognitive layer causing learners to overestimate their learning performance and to overlook gaps on the content layer. This effect may explain overestimation of learning performance in the first study trial. However, when restudying items, knowledge on the content layer should already be activated by the first study trial. This leads to an increased probability of resonant states in sensory memory on the content layer. This increased probability of resonant states in turn increases the probability that restudying items contributes to learning, without learners being aware of this fact. As an effect, learners do not recognize their improvement. Thus, the concept of resonant states explains why learners improve in content learning through restudy much more than they are aware of; consequently, their learning performance increases much more than their JOLs, resulting in the UWP effect. 


\section{Findings on the Expertise Reversal Effect}

One typical finding of research on cognitive load is the so-called "expertise reversal effect" (e.g., Kalyuga 2007; Kalyuga and Renkl 2010). The expertise reversal effect posits that instructional aids effective for low-knowledge learners do not improve but rather hinder the learning of high-knowledge learners. For example, Arslan-Ari (2013) investigated the effects of cueing (labels and pictures) and prior knowledge on learning and mental effort in a multimedia learning environment. The results indicate that, not surprisingly, high-knowledge learners scored higher on a retention test than lowknowledge learners. However, surprisingly, high-knowledge learners also reported higher mental effort. In addition, they did not show superior performance in deep learning as assessed by transfer and matching tasks. Kalyuga et al. (1998) conducted longitudinal studies to investigate the interaction of learners' different expertise levels and the efficiency of instructional formats. Results indicate that instructions that were beneficial for novice learners (Exp. 1) became ineffective or even had negative consequences on performance and mental effort (Exp. 3) when learners' expertise increased. Another example was reported by Nückles et al. (2010). They examined the expertise reversal effect during journal writing. In their first study, the experimental group received a combination of cognitive and metacognitive prompts, whereas the control group did not receive any prompts. First, these instructional aids led to an improvement of the experimental group over the control group, but over time this advantage diminished. To avoid this expertise reversal effect, Nückles et al. used a fading-out prompts procedure in a second experiment. The fading-out group was compared with a group that received permanent prompts. Results indicate that the fading-out group reported significantly more cognitive strategies and showed higher learning outcomes than the permanent prompts group.

This exemplary collection of studies has demonstrated that the expertise reversal effect is sensitive to instructional aids presented on all three layers, e.g., cues highlighting task requirements (Arslan-Ari 2013; Kalyuga et al. 1998) and cues in the form of learning strategy affordances and metacognitive cues (Nückles et al. 2010). Additionally, these studies have examined the expertise reversal effect with regard to learning as well as mental effort (that is, consciously invested working memory capacity). Based on the interactive layers model, we assume that the same mechanism helps to explain the expertise reversal effect on all three layers. When learners possess low prior knowledge, instructional aids strengthen certain information of the learning task as well as certain long-term memory information, allowing for their transfer to working memory without amplification by resonant states in sensory memory. As a result, learners focus on this relevant information when consciously constructing schemes. However, when learners possess high prior knowledge, information not supported by instructional aids has a high probability of creating resonant states in sensory memory and of activating individuals' prior knowledge on all layers. If information strengthened by instructional aids does not match activated prior knowledge, it interferes with already created resonant states. This interference prevents resonant states from automatically contributing to learning. Instead, resonant states as well as information strengthened by instructional aids are transferred to working memory, and learners must invest working memory capacity to solve the conflict between information strengthened by instructional aids and information amplified by resonant states. 


\section{Validating the Interactive Layers Model}

The interactive layers model of self-regulation and cognitive load suggests a new interpretation of existing empirical findings, as demonstrated in the previous section. However, of course, other models suggest different re-interpretations, and future research is needed to test which of the different possible re-interpretations is the most valid. Thus, the new interpretations of existing data do not validate the interactive layers model. For validation purposes, novel hypotheses should be derived from the model and tested empirically. In this section, we suggest a novel hypothesis derived from the model, and we elaborate on a possible study design. However, conducting the respective study would be part of future research.

The most innovative (or challenging) part of our model may be the assumption of resonant states and, in combination with resonant states, the assumption of unconscious self-regulation. Therefore, we have concentrated on generating a hypothesis concerning unconscious selfregulation of a learning strategy and focusing on the learning strategy layer. On the learning strategy layer, resonant states result from learning strategy affordances that match activated learning strategy knowledge. If learning strategy affordances meet expectations that build on learning strategy knowledge, learning strategy use has a high probability of being unconscious and not inducing cognitive load. Given that self-regulated learners have sufficiently practiced a certain learning strategy, the model suggests that the use of this strategy is likely to be unconscious as long as the learning task can successfully be solved with this learning strategy and as long as affordances trigger this strategy. However, as soon as learners experience obstacles when using the learning strategy - for example, expectations are not met anymorelearning strategy use should become conscious, with cognitive processes of the learning strategy performed in working memory inducing cognitive load.

Investigating this hypothesis requires measurement of whether learning strategy use is performed consciously or unconsciously. Conscious learning strategy use can be assessed by any type of verbal data, such as think-aloud protocols (Ericsson and Simon 1980; Sonnenberg and Bannert 2019). In contrast, unconscious learning strategy application cannot be verbalized since learners are not aware of using a learning strategy. Thus, unconscious learning strategy use only expresses itself in learning strategy behavior, as with eye movements when reading. As a consequence, in cases where self-regulated learners show a certain learning strategy behavior without being able to verbally report the learning strategy use, learning strategy use is unconscious, but as soon as learners can and do report learning strategy use, it is conscious.

In order to test whether learning strategy use is unconscious when learning strategy affordances meet expectations and becomes conscious as soon as learners experience obstacles, one might consider the previously mentioned example of a text with highlighted words. These highlighted words are affordances for the learning strategy of skimming the text and using highlighted words to activate focused prior knowledge. This highlighted-word affordance matches expectations derived from learning strategy knowledge, allowing the skimming strategy to be unconscious. But if some or all of the highlighted words are randomly highlighted, learners would experience an obstacle and the use of the skimming strategy would become conscious.

One could test this analysis asking learners who are sufficiently capable of using the skimming strategy to read a text with highlighted words. Learning strategy use could simultaneously be assessed by a behavior-based measure, such as eye-tracking and think-aloud protocols (e.g., Trevors et al. 2016). One would expect that, when highlighted words activate relevant prior knowledge, learners skim more or less fluently the highlighted words without 
verbally reporting to do so. In other words, behavioral data and verbal data would differ. As soon as learners come to words that are highlighted for an unknown reason, though, they experience an obstacle. One would then expect this to lead to disturbances in the eye-tracking data as well as verbal reports on the skimming strategy in the think-aloud protocols.

\section{Conclusion}

In this paper, we proposed an interactive layers model of self-regulated learning and cognitive load. It is a descriptive model in that it shows different paths of information processing without prescribing one ideal path of information processing or of learning. The model builds on assumptions derived from "classical" models of information processing (Atkinson and Shiffrin 1968; Baddeley and Hitch 1974), skill acquisition (Anderson 1982), self-regulated learning (Boekaerts 1997; Winne and Hadwin 1998; Zimmerman 2008), and metamemory (Nelson and Narens 1990). Additionally, it adds a mechanism derived from the Adaptive Resonance Theory (Grossberg 1976a, b, 1999, 2019)—namely, resonant states-which emphasizes the important role of sensory memory in self-regulated learning. We assume that self-regulated learning includes unconscious self-regulation as well as conscious self-regulation processes. Unconscious self-regulation relies on resonant states that automatically and unconsciously contribute to learning. Since resonant states are located in sensory memory, they do not induce cognitive load on working memory. By including resonant states, our model emphasizes the role of sensory memory for learning. Since cognitive load theory has gained increasing relevance, research on self-regulated learning and learning with multimedia has developed a strong focus on working memory and long-term memory, thereby nearly ignoring processes of sensory memory. However, according to dual processing theories (e.g., Evans 2006; Kahneman 2003; Sloman 1996), processes in sensory memory are crucial for learning. Theorizing these processes, therefore, is helpful for explaining the effects of self-regulated learning and cognitive load and for explaining how self-regulation does induce cognitive load (in cases of conscious and intentional self-regulation) or does not (in cases of unconscious and automatic self-regulation).

We have demonstrated the usefulness of the model by interpreting empirical findings reported in the literature of self-regulated learning, metamemory research, and cognitive load theory, with theoretical assumptions derived from the interactive layers model. Of course, these findings are just exemplary, and we guess that more inconsistent findings can be found in the literature that our model helps to explain. Additionally, we assume that there exist many empirical findings that have not yet been published because they were contrary with what the authors of the studies expected based on the theories they used. Perhaps the interactive layers model can lay theoretical ground for re-analyzing these unpublished data.

Funding Open Access funding provided by Projekt DEAL.

Open Access This article is licensed under a Creative Commons Attribution 4.0 International License, which permits use, sharing, adaptation, distribution and reproduction in any medium or format, as long as you give appropriate credit to the original author(s) and the source, provide a link to the Creative Commons licence, and indicate if changes were made. The images or other third party material in this article are included in the article's Creative Commons licence, unless indicated otherwise in a credit line to the material. If material is not included in the article's Creative Commons licence and your intended use is not permitted by statutory regulation or exceeds the permitted use, you will need to obtain permission directly from the copyright holder. To view a copy of this licence, visit http://creativecommons.org/licenses/by/4.0/. 


\section{References}

Abelson, R. P. (1981). Psychological status of the script concept. American Psychologist, 36(7), 715-729. https://doi.org/10.1037/0003-066X.36.7.715.

Aghaie, R., \& Zhang, L. J. (2012). Effects of explicit instruction in cognitive and metacognitive reading strategies on Iranian EFL students' reading performance and strategy transfer. Instructional Science, 40(6), 10631081.

Anderson, J. R. (1982). Acquisition of cognitive skill. Psychological Review, 89(4), 369-406.

Arslan-Ari, I. (2013). Examining the effects of cueing and prior knowledge on learning, mental effort, and study time in a complex animation. Unpublished dissertation. Texas Tech University.

Atkinson, R. C., \& Shiffrin, R. M. (1968). Human memory: A proposed system and its control processes. In K. Spence (Ed.), The psychology of learning and motivation (Vol. 2, pp. 89-195). Academic Press.

Azevedo, R., Moos, D. C., Greene, J. A., Winters, F. I., \& Cromley, J. G. (2008). Why is externally-facilitated regulated learning more effective than self-regulated learning with hypermedia? Educational Technology Research and Development, 56(1), 45-72. https://doi.org/10.1007/s11423-007-9067-0.

Baddeley, A. D., \& Hitch, G. (1974). Working memory. In G. H. Bower (Ed.), The psychology of learning and motivation (Vol. 22, pp. 139-191). Academic Press.

Baker, L. (1994). Fostering metacognitive development. In H. W. Reese (Ed.), Advances in child development and behavior (Vol. 25, pp. 201-239). Academic Press.

Bartlett, F. (1932). Remembering: A study in experimental and social psychology. Cambridge University Press.

Berry, D. C., \& Dienes, Z. (1993). Implicit learning: Theoretical and empirical issues. Lawrence Erlbaum.

Blickle, G. (1996). Personality traits, learning strategies, and performance. European Journal of Personality, 48, 266-279.

Boekaerts, M. (1997). Self-regulated learning: A new concept embraced by researchers, policy makers, educators, teachers, and students. Learning and Instruction, 7(2), 161-186.

Bor, D., \& Seth, A. K. (2012). Consciousness and the prefrontal parietal network: Insights from attention, working memory, and chunking. Frontiers in Psychology, 3, 1-14. https://doi.org/10.3389 /fpsyg.2012.00063.

Broadbent, D. E., Fitz Gerald, P., \& Broadbent, M. H. P. (1986). Implicit and explicit knowledge in the control of complex systems. British Journal of Psychology, 77(1), 33-50.

Butler, D. L., \& Winne, P. H. (1995). Feedback and self-regulated learning: A theoretical synthesis. Review of Educational Research, 65(3), 245-281.

Chi, M., Glaser, R., \& Rees, E. (1982). Expertise in problem solving. In R. Sternberg (Ed.), Advances in the psychology of human intelligence (pp. 7-75). Lawrence Erlbaum.

Churchill, E. F., \& Gilmore, D. J. (1998). Selection through rejection: Reconsidering the invariant learning paradigm. The Quarterly Journal of Experimental Psychology A: Human Experimental Psychology, 51A(1), 1-17. https://doi.org/10.1080/027249898391738.

Cleeremans, A. (1993). Mechanisms of implicit learning: Connectionist models of sequence processing. MIT Press.

Cohen, M. A., Cavanagh, P., Chun, M. M., \& Nakayama, K. (2012). The attentional requirements of consciousness. Trends in Cognitive Science, 16(8), 411-417.

Cowan, N. (1997). Attention and memory: An integrated framework. Oxford University Press.

Cowan, N. (2008). Learning and memory: A comprehensive reference. In J. H. Byrne (Ed.), Learning and memory: A comprehensive reference. Elsevier.

De Boer, H., Donker, A. S., Kostons, D., \& van der Werf, G. (2018). Long-term effects of metacognitive strategy instruction on student academic performance: A meta-analysis. Educational Research Review, 24, 98-115.

De Lisi, R. (1987). A cognitive-developmental model of planning. In S. L. Friedman, E. F. Scholnick, \& R. R. Cocking (Eds.), Blueprints for thinking: The role of planning in cognitive development (pp. 79-109). Cambridge: Cambridge University Press.

Dienes, Z., \& Berry, D. C. (1997). Implicit learning: Below the subjective threshold. Psychonomic Bulletin Review, 4(1), 3-23.

Dunlosky, J., \& Nelson, T. O. (1992). Importance of the kind of cue for judgments of learning (JOL) and the delayed-JOL effect. Memory \& Cognition, 20(4), 374-380.

Eilers, L. H., \& Pinkley, C. (2006). Metacognitive strategies help students to comprehend all text. Reading Improvement, 43, 13-29.

Engelschalk, T., Steuer, G., \& Dresel, M. (2017). Quantity and quality of motivational regulation among university students. Educational Psychology, 37, 1154-1170.

Ericsson, K. A., \& Simon, H. (1980). Verbal repots as data. Psychological Review, 87(3), 215-251.

Evans, J. S. B. T. (2006). The heuristic-analytic theory of reasoning: Extension and evaluation. Psychonomic Bulletin and Review, 13(3), 378-395. 
Flavell, J. H. (1979). Metacognition and cognitive monitoring: A new area of cognitive-developmental inquiry. American Psychologist, 34(10), 906-911.

Frensch, P. A., \& Rünger, D. (2003). Implicit learning. Current Directions in Psychological Science, 12(1), 1318. https://doi.org/10.1111/1467-8721.01213.

Glogger, I., Schwonke, R., Holzäpfel, L., Nückles, M., \& Renkl, A. (2012). Learning strategies assessed by journal writing: Prediction of learning outcomes by quantity, quality, and combinations of learning strategies. Journal of Educational Psychology, 104(2), 452-468. https://doi.org/10.1037/a0026683.

Grossberg, S. (1976a). Adaptive pattern classification and universal recoding. I. Parallel development and coding of neural feature detectors. Biological Cybernetics, 23(3), 121-134.

Grossberg, S. (1976b). Adaptive pattern classification and universal recoding. II. Feedback, expectation, olfaction, and illusions. Biological Cybernetics, 23(4), 187-202.

Grossberg, S. (1999). The link between brain learning, attention, and consciousness. Consciousness and Cognition, 8(1), 1-44.

Grossberg, S. (2019). The resonant brain: How attentive conscious seeing regulations action sequences that interact attentive cognitive learning, recognition, and prediction. Attention, Perception, \& Psychophysics, 81(7), 2237-2264.

Hayes, N. A., \& Broadbent, D. E. (1988). Two modes of learning for interactive tasks. Cognition, 28(3), 249276.

Kahneman, D. (2003). A perspective on judgment and choice: Mapping bounded rationality. American Psychologist, 58(9), 697-720.

Kalyuga, S. (2007). Expertise reversal effect and its implications for learner-tailored instruction. Educational Psychology Review, 19(4), 509-539.

Kalyuga, S., \& Renkl, A. (2010). Expertise reversal effect and its instructional implications: Introduction to the special issue. Instructional Science, 38(3), 209-215.

Kalyuga, S., Chandler, P., \& Sweller, J. (1998). Levels of expertise and instructional design. Human Factors, 40(1), 1-17.

Kintsch, W. (1998). Comprehension: A paradigm for cognition. Cambridge University Press.

Koedinger, K., \& Anderson, J. R. (1990). Abstract planning and perceptual chunks: Elements of expertise in geometry. Cognitive Science, 14(4), 511-550.

Koedinger, K. R., Corbett, A. T., \& Perfetti, C. (2012). The knowledge-learning-instruction framework: Bridging the science-practice chasm to enhance robust student learning. Cognitive Science, 36(5), 757-798. https://doi.org/10.1111/j.1551-6709.2012.01245.x.

Komori, M. (2016). Effects of working memory capacity on metacognitive monitoring: A study of group differences using listening span test. Frontiers in Psychology, 7, 285. https://doi.org/10.3389 /fpsyg.2016.00285.

Koriat, A. (1997). Monitoring one's own knowledge during study: A cue-utilization approach to judgments of learning. Journal of Experimental Psychology: General, 126(4), 349-370.

Koriat, A. (2007). Metacognition and consciousness. In P. D. Zelazo, M. Moscovitch, \& E. Thompson (Eds.), The Cambridge handbook of consciousness (pp. 289-326). Cambridge University Press.

Koriat, A., Sheffer, L., \& Ma'ayan, H. (2002). Comparing objective and subjective learning curves: Judgements of learning exhibit increased underconfidence with practice. Journal of Experimental Psychology: General, $131(2), 147-162$.

Koriat, A., Nussinson, R., \& Ackerman, R. (2014). Judgements of learning depend on how learners interpret study effort. Journal of Experimental Psychology: Learning, Memory, and Cognition, 40, $1624-1637$.

Kuldas, S., Ismail, H. N., Hashim, S., \& Bakar, Z. A. (2013). Unconscious learning processes: Mental integration of verbal and pictorial instructional materials. SpringerPlus, 2(1), 105. https://doi.org/10.1186/2193-1801-2105.

Leopold, C., \& Leutner, D. (2002). Der Einsatz von Lernstrategien in einer konkreten Lernsituation bei Schülern unterschiedlicher Jahrgangsstufen [Use of learning strategies in a concrete learning situation with students in different grades]. Zeitschrift für Pädagogik, 45, 240-258.

Leopold, C., \& Leutner, D. (2015). Improving students' science text comprehension through metacognitive selfregulation when applying learning strategies. Metacognition and Learning, 10(3), 313-346.

Mayer, R. E. (1997). Multimedia learning: Are we asking the right questions? Educational Psychologist, 32(1), $1-19$.

Mazzioni, G., Cornoldi, C., \& Marchitelli, G. (1990). Do memorability ratings affect study-time allocation? Memory \& Cognition, 18(2), 196-204.

Nelson, T. O. (1996). Consciousness and metacognition. American Psychologist, 51(2), 102-116.

Nelson, T. O., \& Narens, L. (1990). Metamemory: A theoretical framework and new findings. In G. H. Bower (Ed.), The psychology of learning and motivation (Vol. 26, pp. 125-173). Academic Press. 
Nückles, M., Hübner, S., \& Renkl, A. (2009). Enhancing self-regulated learning by writing learning protocols. Learning and Instruction, 19(3), 259-271.

Nückles, M., Hübner, S., Dümer, S., \& Renkl, A. (2010). Expertise reversal effects in writing-to-learn. Instructional Science, 38(3), 237-258.

Panadero, E. (2017). A review of self-regulated learning: Six models and four directions for research. Frontiers of Psychology, 8, 193-220.

Paris, S. G., Lipson, M. Y., \& Wixson, K. K. (1983). Becoming a strategic reader. Contemporary Educational Psychology, 8(3), 293-316.

Pintrich, P. R., \& De Groot, E. V. (1990). Motivational and self-regulated learning components of class-room academic performance. Journal of Educational Psychology, 82(1), 33-40.

Pintrich, P. R., \& Garcia, T. (1993). Intraindividual differences in students' motivation and self-regulated learning. Zeitschrift für Pädagogische Psychologie, 7, 99-107.

Pintrich, P. R., Smith, D. A. F., Garcia, T., \& McKeachie, W. J. (1993). Reliability and predictive validity of the motivated strategies for learning questionnaire (MSLQ). Educational and Psychological Measurement, 53(3), 801-813.

Pitts, M. A., Lutsyshyna, L. A., \& Hillyard, S. A. (2018). The relationship between attention and consciousness: An expanded taxonomy and implications for 'no-report' paradigms. Philosophical Transactions of the Royal Society $B, 373,1-12$.

Pressley, M., Borkowski, J. G., \& Schneider, W. (1987). Cognitive strategies: Good strategies users coordinate metacognition and knowledge. In R. Vasta \& G. Whitehurst (Eds.), Annals of child development (Vol. 4, pp. 89-129). JAI Press.

Pressley, M., Borkowski, J. G., \& Schneider, W. (1989). Good information processing: What it is and how education can promote it. International Journal of Educational Research, 13(8), 857-867.

Puustinen, M., \& Pulkkinen, L. (2001). Models of self-regulated learning: A review. Scandinavian Journal of Educational Research, 45(3), 269-286.

Reber, A. S. (1967). Implicit learning of artificial grammars. Journal of Verbal Learning and Verbal Behavior, $6(6), 855-863$.

Reber, A. S., Walkenfeld, F. F., \& Hernstadt, R. (1991). Implicit and explicit learning: Individual differences and IQ. Journal of Experimental Psychology: Learning, Memory, and Cognition, 17(5), 888-896. https://doi. org/10.1037/0278-7393.17.5.888.

Reder, L. M. (1996). Implicit memory and metacognition. Lawrence Erlbaum Associates.

Rhodes, S., \& Cowan, N. (2018). Attention in working memory: Attention is needed but it yearns to be free. Annals of the New York Academy of Science, 1424(1), 52-63.

Rogiers, A., Merchie, E., Van Keer, H. (2020). What they say is what they do? Comparing task-specific selfreports, think-aloud protocols, and study traces for measuring secondary school students' text-learning strategies. European Journal of Psychology of Education, 35, 315-332.

Runeson, S., Juslin, P., \& Olsson, H. (2000). Visual perception of dynamic properties: Cue heuristics versus direct-perceptual competence. Psychological Review, 107(3), 525-555.

Samuels, S. J., Ediger, K. A. M., Willcutt, J. R., \& Palumbo, T. (2005). Role of automaticity in metacognition and literacy instruction. In S. E. Israel, K. L. Bauserman, C. C. Block, \& K. Kinnucan-Welsch (Eds.), Metacognition in literacy learning: Theory, assessment, instruction and professional development (pp. 4259). Lawrence Erlbaum Associates.

Schneider, W., \& Pressley, M. (1989). Good strategy use: A general model, a specific example, and comments on how to do research on the development of strategy proficiency. In W. Schneider \& M. Pressley (Eds.), Memory development between 2 and 20 (pp. 121-159). Springer.

Sloman, S. A. (1996). The empirical case for two systems of reasoning. Psychological Bulletin, 119(1), 3-22.

Son, L. K., \& Metcalfe, J. (2000). Metacognitive and control strategies in study-time allocation. Journal of Experimental Psychology: Learning, Memory \& Cognition, 26, 204-221.

Sonnenberg, C., \& Bannert, M. (2019). Using process mining to examine the sustainability of instructional support: How stable are the effects of metacognitive prompting on self-regulatory behavior. Computers in Human Behavior, 96, 259-272.

Stadler, M. A. (1992). Statistical structure and implicit serial learning. Journal of Experimental Psychology: Learning, Memory, and Cognition, 18(2), 318-327. https://doi.org/10.1037/0278-7393.18.2.318.

Stadler, M. A., \& Frensch, P. A. (Eds.). (1998). Handbook of implicit learning. Sage.

Stanley, W. B., Mathews, R. C., Buss, R. R., \& Kotler-Cope, S. (1989). Insight without awareness: On the interaction of verbalization, instruction and practice in a simulated process control task. The Quarterly Journal of Experimental Psychology A: Human Experimental Psychology, 41(3-A), 553-577. https://doi. org/10.1080/14640748908402382.

Süß, H.-M. (1996). Intelligenz, Wissen und Problemlösen [Intelligence, knowledge, and problem solving]. Hogrefe. 
Sweller, J. (1988). Cognitive load during problem solving: Effects on learning. Cognitive Science, 12(2), 257285.

Sweller, J., van Merrienboer, J. J. G., \& Paas, F. G. W. C. (1998). Cognitive architecture and instructional design. Educational Psychology Review, 10(3), 251-296.

Sweller, J., van Merrienboer, J. J. G., \& Paas, F. (2019). Cognitive architecture and instructional design: 20 years later. Educational Psychology Review, 31, 261-292.

Thompson, V. A. (2009). Dual-process theories: A metacognitive perspective. In J. Evans \& K. Frankish (Eds.), In two minds: dual processes and beyond (pp. 171-195). Oxford University Press.

Thompson, V. A., Prowse Turner, J. A., \& Pennycook, G. (2011). Intuition, metacognition, and reason. Cognitive Psychology, 63(3), 107-140.

Trevors, G., Feyzi-Behnagh, R., Azevedo, R., \& Bouchet, F. (2016). Self-regulated learning processes vary as a function of epistemic beliefs and contexts: Mixed method evidence from eye tracking and concurrent and retrospective reports. Learning and Instruction, 42, 31-46.

Valcke, M. (2002). Cognitive load: Updating the theory? Learning and Instruction, 12(1), 147-154. https://doi. org/10.1016/S0959-4752(01)00022-6.

Veenman, M. V. J., Prins, F. J., \& Elshout, J. J. (2002). Initial learning in a complex computer simulated environment: The role of metacognitive skills and intellectual ability. Computers in Human Behavior, 18(3), $327-342$.

Veenman, M. V. J., Kok, R., \& Blote, A. W. (2005). The relationship between intellectual and metacognitive skills in early adolescence. Instructional Science, 33(3), 193-211.

Veenman, M. V. J., Van Hout-Wolters, B. H. A. M., \& Afflerbach, P. (2006). Metacognition and learning: Conceptual and methodological considerations. Metacognition and Learning, 1(1), 3-14.

Waldeyer, J., Fleischer, J., Wirth, J., \& Leutner, D. (2019). Validating the resource-management inventory (ReMI): Testing measurement invariance and predicting academic achievement in a sample of first-year university students. European Journal of Psychological Assessment. Online pre-publication., 1-10. https://doi.org/10.1027/1015-5759/a000557.

Wang, M. C., Haertel, G. D., \& Walberg, H. J. (1990). What influences learning? A content analysis of review literature. Journal of Educational Research, 84(1), 30-43.

Weinert, F. E. (1982). Selbstgesteuertes Lernen als Voraussetzung, Methode und Ziel des Unterrichts [Selfdirected learning as a pre-requisite, method, and goal of teaching]. Unterrichtswissenschaft, 2, 99-110.

Weinstein, C. E., \& Mayer, R. E. (1986). The teaching of learning strategies. In M. Wittrock (Ed.), The handbook of research on teaching (pp. 315-327). Macmillan.

Winne, P. H. (1996). A metacognitive view of individual differences in self-regulated learning. Learning and Individual Differences, 8(4), 327-353.

Winne, P. H., \& Hadwin, A. F. (1998). Studying as self-regulated learning. In D. J. Hacker, J. Dunlosky, \& A. C. Graesser (Eds.), Metacognition in educational theory and practice (pp. 277-304). Lawrence Erlbaum Associates.

Winne, P. H., \& Perry, N. (2000). Measuring self-regulated learning. In M. Boekaerts, P. Pintrich, \& M. Zeidner (Eds.), Handbook of self-regulation (pp. 531-566). Academic Press.

Wirth, J. (Guest Ed.) (2009). Promoting self-regulated learning through prompts. Zeitschrift für Pädagogische Psychologie, 23, 91-145, 2.

Wirth, J., \& Leutner, D. (2008). Self-regulated learning as a competence: Implications of theoretical models for assessment methods. Zeitschrift für Psychologie/Journal of Psychology, 216(2), 102-110.

Wolters, C. A. (1998). Self-regulated learning and college students' regulation of motivation. Journal of Educational Psychology, 90(2), 224-235.

Zimmerman, B. J. (2000). Attaining self-regulation: A social cognitive perspective. In M. Boekaerts, P. R. Pintrich, \& M. Zeidner (Eds.), Handbook of self-regulation (pp. 13-39). Academic Press.

Zimmerman, B. J. (2008). Theories of self-regulated learning and academic achievement: An overview and analysis. In B. J. Zimmerman \& D. H. Schunk (Eds.), Self-regulated learning and academic achievement: Theoretical perspectives (2nd ed., pp. 1-36). Lawrence Erlbaum Associates.

Publisher's Note Springer Nature remains neutral with regard to jurisdictional claims in published maps and institutional affiliations. 